Contributions to Game Theory and Management, XIII, 244-251

\title{
Feedback Based Strategies for Autonomous Linear Quadratic Cooperative Differential Games with Continuous Updating*
}

\author{
Ildus Kuchkarov \\ St.Petersburg State University, \\ Faculty of Applied Mathematics and Control Processes, \\ Universitetskaya emb. 7/9, St.Petersburg, 198504, Russia \\ E-mail: kuchkarov_ildus@mail.ru
}

\begin{abstract}
In the paper the class of linear quadratic cooperative differential games with continuous updating is considered. Here the case of feedback based strategies is used to construct cooperative strategies with continuous updating. Characteristic function with continuous updating, cooperative trajectory with continuous updating and cooperative solution are constructed. For the cooperative solution we use the Shapley value.
\end{abstract}

Keywords: cooperative differential games; differential games with continuous updating; differential games with continuous updating.

\section{Introduction}

Most of the real-life conflict-driven processes evolve continuously in time, and their participants continuously receive updated information and adapt. Main models considered in the classical differential game theory are associated with problems defined on a fixed time interval (Kleimenov, 1993), problems defined on an infinite time interval with discounting (Basar and Olsder, 1995), problems defined on a random time interval (Shevkoplyas, 2014). One of the first works in the theory of differential games is devoted to the differential pursuit game (Petrosyan and Murzov, 1966). In all the above models and approaches it is assumed that at the beginning of the game players know all information about the game dynamics and player's preferences. However, these approaches do not take into account the fact that in many real conflict-controlled processes, players at the initial time instant do not have all information about the game. Therefore classical approaches for defining in some sense optimal strategies (for example, Nash equilibrium), such as Hamilton-Jacobi-Bellman equation (Bellman, 1957) or the Pontryagin maximum principle (Pontryagin, 1966), cannot be directly used to construct a large range of real game-theoretic models.

Most conflict-driven processes in real-life evolve continuously in time, and their participants continuously receive updated information and adapt accordingly. This paper is dedicated to the new class of differential games with continuous updating, in particular, the cooperative setting of an autonomous case is considered. In the game models with continuous updating, it is assumed that players have information about motion equations and cost functions only on $[t, t+\bar{T}]$, where $\bar{T}$ is information horizon, $t$ is current time instant, receive updated information regarding motion equations and cost functions as time $t \in\left[t_{0},+\infty\right)$ evolves.

In the framework of the dynamic updating approach, the following papers were published (Petrosian, 2016a; Petrosian, 2016b). Their author laid a foundation for

\footnotetext{
* Research was supported by a grant from the Russian Science Foundation (Project No 18-71-00081).
} 
further study of a class of games with dynamic updating. It is assumed that the information about motion equations and payoff functions is updated in discrete time instants and interval on which players know the information is defined by the value of the information horizon. However, the class of games with continuous updating provides with the new theoretical results. The class of differential games with continuous updating was considered in the papers (Kuchkarov and Petrosian, 2019; Petrosian and Tur, 2019), here it is supposed that the updating process evolves continuously in time. In the paper (Petrosian and Tur, 2019), the system of HamiltonJacobi-Bellman equations are derived for the Nash equilibrium in a game with continuous updating. In the paper (Kuchkarov and Petrosian, 2019) the class of linear-quadratic differential games with continuous updating is considered and the explicit form of the Nash equilibrium is derived.

In this paper, the results of the paper (Kuchkarov and Petrosian, 2019) is extended. It is interesting to construct not only the non-cooperative solution but also to obtain the form of cooperative strategies, characteristic function and cooperative solution for continuous updating case, i.e. to study cooperative differential game model. The main contribution of this paper lies in the cooperative setting and consideration of an autonomous case when motion equations and cost functions do not explicitly depend on the time parameter.

The paper is structured as follows. In section 2., a description of game model with continuous updating is presented. Section III is devoted to cooperative strategies with continuous updating. In section IV, the explicit form of characteristic function and Shapley value for the linear quadratic autonomous game model are presented. Section V presents our conclusions.

\section{Linear Quadratic Autonomous Game Model with Continuous Updating}

Consider $n$-player differential game $\Gamma(x, t, \bar{T})$, defined on the interval $[t, t+\bar{T}]$, where $t \in\left[t_{0},+\infty\right), 0<\bar{T}<+\infty$.

Motion equations of $\Gamma(x, t, \bar{T})$ have the form

$$
\dot{x}^{t}(s)=A x^{t}(s)+B_{1} u_{1}^{t}\left(s, x^{t}\right)+\ldots+B_{n} u_{n}^{t}\left(s, x^{t}\right), \quad x^{t}(t)=x,
$$

where $x^{t} \in \mathbb{R}^{l}, u^{t}=\left(u_{1}^{t}, \ldots, u_{n}^{t}\right), u_{i}^{t}=u_{i}^{t}\left(s, x^{t}\right) \in \mathbb{R}^{k}, t \in\left[t_{0},+\infty\right)$.

Cost function of player $i \in N$ in the game $\Gamma(x, t, \bar{T})$ is defined as

$$
K_{i}^{t}\left(x^{t}, t, \bar{T} ; u^{t}\right)=\int_{t}^{t+\bar{T}}\left(\left(x^{t}(s)\right)^{\prime} Q_{i} x^{t}(s)+\sum_{j=1}^{n}\left(u_{j}^{t}\left(s, x^{t}\right)\right)^{\prime} R_{i j} u_{j}^{t}\left(s, x^{t}\right)\right) d s,
$$

where $Q_{i}, R_{i j}$ are assumed to be symmetric, $R_{i j}$ are positive semi-defined, $R_{i i}$ is positive defined, $(\cdot)^{\prime}$ means transpose here and hereafter and $x^{t}(s), u^{t}(s, x)$ are trajectory and strategies in the game $\Gamma(x, t, \bar{T})$. Player $i$ is minimizing (2).

Differential game with continuous updating evolves according to the rule:

Time parameter $t \in\left[t_{0},+\infty\right)$ evolves continuously, as a result players continuously receive updated information about motion equations and cost functions under $\Gamma(x, t, \bar{T})$.

Strategies $u(t, x)$ in the game model with continuous updating are defined in the following way:

$$
u(t, x)=u^{t}(t, x), t \in\left[t_{0},+\infty\right),
$$


where $u^{t}(s, x), s \in[t, t+\bar{T}]$ are some fixed strategies defined in the subgame $\Gamma(x, t, \bar{T})$.

State $x(t)$ in the model with continuous updating is defined according to

$$
\dot{x}(t)=A x(t)+B_{1} u_{1}(t, x)+\ldots+B_{n} u_{n}(t, x),
$$

$x\left(t_{0}\right)=x_{0}, x \in \mathbb{R}^{l}$, with strategies with continuous updating $u(t, x)$ involved.

The essential difference between the game model with continuous updating and classic differential game $\Gamma\left(x_{0}, T-t_{0}\right)$ with prescribed duration is that players in the initial game are guided by the costs that they will eventually receive on the interval $\left[t_{0}, T\right]$, but in the case of a game with continuous updating, at the time instant $t$ they orient themselves on the expected costs (2), which are calculated using information about the game structure defined on the interval $[t, t+\bar{T}]$.

\section{Optimal Cooperative Strategy for Games with Continuous Updating}

In a game with continuous updating, the player at each moment focuses on the nearest event horizon, trying to maximize profits on it. The player chooses the optimal strategy for, generally speaking, a new game at each moment in time. And the optimal strategy for the whole game is made up of each particular decision.

For example, consider two time intervals $[t, t+\bar{T}]$ and $[t+\epsilon, t+\bar{T}+\epsilon], \epsilon<<\bar{T}$. According to the problem statement, $u^{*}(t, x)$ at the instant $t$ should coincide with the optimal cooperative strategy in the game defined on the interval $[t, t+\bar{T}]$ and $u^{*}(t+\epsilon, x)$ at instant $t+\epsilon$ should coincide with the optimal cooperative strategy in the game defined on the interval $[t+\epsilon, t+\epsilon+\bar{T}]$. Therefore direct application of classical approaches for determining optimal cooperative strategy in feedback strategies is not possible.

In cooperative case all players minimize one functional

$$
K^{t}\left(x^{t}, t, \bar{T} ; u^{t}\right)=\sum_{i \in N} K_{i}^{t}\left(x^{t}, t, \bar{T} ; u^{t}\right),
$$

where $K_{i}^{t}\left(x^{t}, t, \bar{T} ; u^{t}\right)$ is defined in (2).

To determine the solution of a game with continuous updating, we introduce the concept of a generalized solution as a combination of solutions in subgames.

Definition 1. Strategy profile $\tilde{u}^{*}(t, s, x)=\left(\tilde{u}_{1}^{*}(t, s, x), \ldots, \tilde{u}_{n}^{*}(t, s, x)\right), t \in\left[t_{0},+\infty\right)$, $s \in[t, t+\bar{T}]$ is a generalized cooperative solution in the game with continuous updating, if for any fixed $t \in\left[t_{0},+\infty\right)$ strategy profile $\tilde{u}^{*}(t, s, x)$ is cooperative solution in the game $\Gamma(x, t, \bar{T}), 0<\bar{T}<\infty$.

Using the generalized cooperative solution it is possible to define a solution concept for a game model with continuous updating.

Definition 2. Strategy profile $u^{*}(t, x)$ is called the cooperative solution with continuous updating, if it is defined in the following way:

$$
u^{*}(t, x)=\left.\tilde{u}^{*}(t, s, x)\right|_{s=t},
$$

where $t \in\left[t_{0},+\infty\right), \tilde{u}^{*}(t, s, x)$ is the generalized cooperative solution defined above. 
Strategy profile $u^{*}(t, x)$ will be used as a solution concept in the cooperative game with continuous updating. Sufficient conditions for the existence of a cooperative solution with continuous updating are presented below.

Theorem 1. For an $N$-person linear-quadratic differential game with $Q_{i} \geq 0$, $R_{i j} \geq 0(i, j \in N), R_{i i}>0(i \in N)$, let there exist a solution set $\left\{Z^{t}, t \geqslant t_{0}\right\}$ to the matrix Riccati differential equations

$$
\begin{aligned}
& \dot{Z}^{t}(\tau)=-\bar{T} A^{\prime} Z^{t}(\tau)-\bar{T} Z^{t}(\tau) A+Z^{t}(\tau) S Z^{t}(\tau)-Q \\
& Z^{t}(1)=0
\end{aligned}
$$

where $\tau \in[0,1], S=\bar{T}^{2} B R^{-1} B^{\prime}, Q=\sum_{i \in N} Q_{i}, B$ and $R$ are block matrices, i.e. $B=\left[B_{1}, \ldots, B_{n}\right], R=\left\{R_{i j}\right\}_{i, j=1}^{n}$.

Then, the differential game with continuous updating admits a cooperative solution with continuous updating given by

$$
u^{*}(t, x)=-R^{-1} B^{\prime} Z^{t}(0) \bar{T} x .
$$

Proof. In order to prove the Theorem we introduce the change of variables.

$$
y^{t}(\tau)=x^{t}(t+\bar{T} \tau), \quad v_{i}^{t}(\tau, y)=u_{i}^{t}(t+\bar{T} \tau, x), \quad i \in N .
$$

By substituting (8) to the motion equations (1), cost function (5) we obtain

$$
\begin{gathered}
\dot{y}^{t}(\tau)=\bar{T} A y^{t}(\tau)+\sum_{i=1}^{N} \bar{T} B_{i} v_{i}^{t}(\tau, y), \\
K^{t}\left(y^{t}, \tau ; v^{t}\right)=\sum_{i \in N} \int_{0}^{1}\left(\left(y^{t}(s)\right)^{\prime} Q_{i} y^{t}(s)+\sum_{j=1}^{N}\left(v_{j}^{t}(s, y)\right)^{\prime} R_{i j} v_{j}^{t}(s, y)\right) d s .
\end{gathered}
$$

The Theorem 5.1 from (Engwerda, 2005) and existence of solution for the system of differential equations ( 7 ) lead to cooperative solution in the subgame $\Gamma(x, t, \bar{T})$ have the form

$$
v^{t, *}(\tau, y)=-R^{-1} B^{\prime} Z^{t}(\tau) \bar{T} y,
$$

Then a generalized cooperative solution in the game with continuous updating has the form

$$
\tilde{u}^{*}(t, s, x)=-R^{-1} B^{\prime} Z_{i}^{t}\left(\frac{s-t}{\bar{T}}\right) \bar{T} x .
$$

Apply the procedure (6) to determine Nash equilibrium with continuous updating using generalized Nash equilibrium (11):

$$
u^{*}(t, x)=-R^{-1} B^{\prime} Z_{i}^{t}(0) \bar{T} x, \quad t \in\left[t_{0},+\infty\right), \quad i \in N .
$$

This proves the theorem.

\section{Cooperative Solution with Continuous Updating}

To determine how to allocate joint cost among the players it is necessary to define how the overall game evolves, how players forecast their behavior at every current time instant $t \in\left[t_{0},+\infty\right]$ for the future interval $[t, t+\bar{T}]$ : forecasted trajectory, characteristic function, a cooperative solution with continuous updating what are the properties. 


\subsection{Characteristic Function for Subgame on Interval $[t, t+\bar{T}]$}

Consider coalition $S$ in $n$-player differential game $\Gamma(x, t, \bar{T})(1)(2)$. The characteristic function is defined as the total cost of the coalition $S$ in the Nash equilibrium $u^{N E}=\left(u_{1}^{N E}, \ldots, u_{n_{S}}^{N E}\right)$ in a game $\Gamma^{S}(x, t, \bar{T})$ with the following set of players: a coalition $S$ acting as one player and players from the set $N \backslash S$ i. e., in the game of $n_{S}=|N \backslash S|+1$ players.

Describe the building of the auxiliary game $\Gamma^{S}(x, t, \bar{T})$. Let the first player of this game be player associated with coalition $S$ for convenience and let other players have been renumbered in some way players from $N \backslash S$. Relabel matrices for $N \backslash S$ players $A^{S}=A, B_{i}^{S}=B_{k_{i}}, Q_{i}^{S}=Q_{k_{i}}, R_{i, j}=R_{k_{i}, k_{j}}$, where $i, j=\overline{2, n_{S}}$ and $i$ is new index in $\Gamma^{S}(x, t, \bar{T})$ of $k_{i}$-th player from $\Gamma(x, t, \bar{T})$. Some matrices for coalition player have a block structure: $B_{1}^{S}=\left[B_{m_{1}} \ldots B_{m_{c}}\right], R_{1,1}^{S}=\left\{R_{i j}\right\}_{i, j=m_{1}}^{m_{c}}$, $R_{i, 1}^{S}=\operatorname{diag}\left(R_{k_{i}, m_{1}}, \ldots, R_{k_{i}, m_{c}}\right)$; other - are sum of corresponding matrices from $\Gamma(x, t, \bar{T}): Q_{1}^{S}=\sum_{m \in S} Q_{m}, R_{1, i}^{S}=\sum_{m \in S} R_{m, k_{i}}$, where $m_{1}, \ldots, m_{c} \in S, i=\overline{2, n_{S}}$.

Thus motion equations of $\Gamma^{S}(x, t, \bar{T})$ have the form

$$
\begin{aligned}
& \dot{x}^{t}(s)=A^{S} x^{t}(s)+B_{1}^{S} u_{1}^{t}\left(s, x^{t}\right)+\ldots+B_{n}^{S} u_{n}^{t}\left(s, x^{t}\right), \\
& x^{t}(t)=x .
\end{aligned}
$$

Cost function of player $i \in N^{S}$ in the game $\Gamma^{S}(x, t, \bar{T})$ is defined as

$$
K_{i}^{S, t}\left(x^{t}, t, \bar{T} ; u^{t}\right)=\int_{t}^{t+\bar{T}}\left(\left(x^{t}(s)\right)^{\prime} Q_{i}^{S} x^{t}(s)+\sum_{j=1}^{n_{S}}\left(u_{j}^{t}\left(s, x^{t}\right)\right)^{\prime} R_{i j}^{S} u_{j}^{t}\left(s, x^{t}\right)\right) d s,
$$

where $x^{t}(s), u^{t}(s, x)$ are trajectory and strategies in the game $\Gamma^{S}(x, t, \bar{T})$.

Lemma 1. For an $N$-person linear-quadratic differential game $\Gamma(x, t, \bar{T})$ with $Q_{i}^{S} \geq$ $0, R_{i j}^{S} \geq 0(i, j \in N), R_{i i}^{S}>0(i \in N)$, let there exist a solution set $\left\{Z_{i}^{S}, i \in N_{S}, t \geqslant\right.$ $\left.t_{0}\right\}$ to the matrix Riccati differential equations

$$
\begin{aligned}
& \frac{d Z_{i}^{S}(\tau)}{d \tau}=-Z_{i}^{S}(\tau) F^{S}(\tau)-\left(F^{S}(\tau)\right)^{\prime} Z_{i}^{S}(\tau)-Q_{i}^{S-} \\
& -\bar{T}^{2} \sum_{j \in N} Z_{j}^{S}(\tau) B_{j}^{S}\left(R_{j j}^{S}\right)^{-1} R_{i j}^{S}\left(R_{j j}^{S}\right)^{-1}\left(B_{j}^{S}\right)^{\prime} Z_{j}^{S}(\tau), \\
& Z_{i}^{S}(1)=0, \quad i \in N,
\end{aligned}
$$

where

$$
F^{S}(\tau)=\bar{T} A^{S}-\bar{T}^{2} \sum_{i \in N} B_{i}^{S}\left(R_{i i}^{S}\right)^{-1}\left(B_{i}^{S}\right)^{\prime} Z_{i}^{S}(\tau) .
$$

Then, the characteristic function for game $\Gamma(x, t, \bar{T})$ has form

$$
\begin{aligned}
V^{t}(S, x, \xi, t+\bar{T}) & =\int_{\xi}^{t+\bar{T}}\left(\left(x^{*}(s, t, x)\right)^{\prime} Q_{1}^{S} x^{*}(s, t, x)+\right. \\
& \left.+\sum_{j=1}^{n}\left(u_{j}^{t}\left(s, x^{*}\right)\right)^{\prime} R_{1 j}^{S} u_{j}^{t}\left(s, x^{*}\right)\right) d s,
\end{aligned}
$$


where $\xi \in[t, t+\bar{T}]$,

$$
u_{i}^{t}(s, x)=-\left(R_{i i}^{S}\right)^{-1}\left(B_{i}^{S}\right)^{\prime} Z_{i}^{S}\left(\frac{s-t}{\bar{T}}\right) \bar{T} x,
$$

$x^{*}(s, t, x)$ is solution of system

$$
\begin{aligned}
\dot{x}^{t}(s) & =A^{S} x^{t}(s)+B_{1}^{S} u_{1}^{t}(s, x)+\ldots+B_{n}^{S} u_{n}^{t}(s, x), \\
x^{t}(t) & =x .
\end{aligned}
$$

Proof. We defined the characteristic function as the total cost of the coalition $S$ in the Nash equilibrium in $\Gamma^{S}(x, t, \bar{T})$.

Similar to theorem 1 proof we use the change of variables (8), the corollary 6.5 from (Basar and Olsder, 1995) and existence of solution for the system of differential equations (13). This implies feedback-based Nash equilibrium strategies in the subgame $\Gamma^{S}(x, t, \bar{T})$ have the form (15).

According to building auxiliary game $\Gamma^{S}(x, t, \bar{T})$ the total cost of the coalition $S$ could be calculated as $K_{1}^{S, t}\left(x^{t}, t, \bar{T}, u^{t}\right)$. Then function (14) is characteristic and system dynamics evolves by (16).

\subsection{Characteristic Function with Continuous Updating}

Consider a game with continuous updating on time interval $\left[t_{0}, T\right]$, where $\bar{T}<<$ $T$. Suppose that the function $\tilde{V}^{t}\left(S ; \tilde{x}_{t}^{*}(s), s, t+\bar{T}\right), S \subseteq N$ is continuously differentiable by $s \in[t, \bar{T}]$ and integrable by $t \in\left[t_{0}, T\right]$. Define characteristic function in game model with continuous updating $V\left(S ; x^{*}(t), t\right)$ in the following way:

Definition 3. Function $V\left(S ; x^{*}(t), t\right), t \in\left[t_{0}, T\right], S \subseteq N$ is a characteristic function with continuous updating, if it is defined as the following integral:

$$
V\left(S ; x^{*}(t), t\right)=\int_{t}^{T}-\left.\frac{d}{d s} \tilde{V}^{\tau}\left(S ; \tilde{x}_{\tau}^{*}(s), s, \tau+\bar{T}\right)\right|_{s=\tau} d \tau,
$$

where $t \in\left[t_{0}, T\right], S \subseteq N, \tilde{V}^{\tau}\left(S ; \tilde{x}_{\tau}^{*}(s), s, t+\bar{T}\right), s \in[\tau, \tau+\bar{T}], \tau \in[t, T), S \subseteq N$ is a characteristic function in the game $\Gamma\left(\tilde{x}_{\tau}^{*}(s), s, \tau+\bar{T}\right)$ defined on the interval $[s, t+\bar{T}]$.

Remark 1. Notice that the integral in (17) can be infinite, if $T=\infty$. Therefore we suppose $T<\infty$.

Theorem 2. For a coalitions $S$ in $N$-person linear-quadratic differential game with $Q_{i}^{S} \geq 0, R_{i j}^{S} \geq 0(i, j \in N), R_{i i}^{S}>0(i \in N)$, let there exist a solution set $\left\{Z_{i}^{S}, i \in N_{S}, t \geqslant t_{0}\right\}$ to the matrix Riccati differential equations (13). Then, the characteristic function with continuous updating has form

$$
V(S, x, t, T)=\int_{t}^{T}\left(x^{*}(s, t, x)\right)^{\prime}\left(Q_{1}^{S}-\bar{T}^{2} \sum_{j=1}^{n_{S}} P_{j}^{\prime} R_{1 j}^{S} P_{j}\right) x^{*}(s, t, x) d s,
$$

where

$$
P_{j}=\left(R_{j j}^{S}\right)^{-1}\left(B_{j}^{S}\right)^{\prime} Z_{j}^{S}(0)
$$


$x^{*}(s, t, x)$ is solution of system

$$
\dot{x}^{t}(s)=\left(A^{S}-\sum_{i=1}^{n_{S}} B_{i}^{S} P_{i}\right) x^{t}(s), \quad x^{t}(t)=x .
$$

Proof. Lemma 1 gives the characteristic function (14) for subgame $\Gamma(x, t, \bar{T})$. By substituting (14) into (17) we get

$$
\begin{gathered}
V(S, x, t, T)=\int_{t}^{T}-\left.\frac{d}{d s} V^{\tau}\left(S ; \tilde{x}_{\tau}^{*}(s), s, \tau+\bar{T}\right)\right|_{s=\tau} d \tau= \\
=\int_{t}^{T}\left(\left(x^{*}(s, t, x)\right)^{\prime} Q_{1}^{S} x^{*}(s, t, x)+\right. \\
\left.+\sum_{j=1}^{n}\left(u_{j}^{s}\left(s, x^{*}(s, t, x)\right)\right)^{\prime} R_{1 j}^{S} u_{j}^{s}\left(s, x^{*}(s, t, x)\right)\right) d s, \\
u_{i}^{s}(s, x)=-\left(R_{i i}^{S}\right)^{-1}\left(B_{i}^{S}\right)^{\prime} Z_{i}^{S}(0) \bar{T} x .
\end{gathered}
$$

By substituting (22) into (21) with (19) we get

$$
\begin{aligned}
V(S, x, t, T) & =\int_{t}^{T}\left(\left(x^{*}(s, t, x)\right)^{\prime} Q_{1}^{S} x^{*}(s, t, x)-\right. \\
& \left.-\bar{T}^{2} \sum_{j=1}^{n}\left(x^{*}(s, t, x)\right)^{\prime} P_{j}^{\prime} R_{1 j}^{S} P_{j} x^{*}(s, t, x)\right) d s .
\end{aligned}
$$

Give (23) in similar terms to (18). Taking into account (22), (16) we can describe system dynamics as (20).

\subsection{Shapley Value with Continuous Updating}

Suppose that all the players united in a coalition of $N$, then, moving along the cooperative trajectory $x^{*}(t)$, they can secure a total cost of $V\left(N, x^{*}(t), t, T\right)$. To determine the costs of each player $i \in N$, we introduce the concept of imputation $\xi\left(x^{*}(t), t, T\right)=\left(\xi_{1}\left(x^{*}(t), t, T\right), \ldots, \xi_{n}\left(x^{*}(t), t, T\right)\right)$, i. e., the cost that the player $i$ will receive after the redistribution of the maximum total win $V\left(N, x^{*}(t), t, T\right)$ between all players.

As a imputation in the game with continuous updating, we will use the Shapley vector:

$$
\begin{aligned}
& S h_{i}\left(x^{*}(t), t, T\right)=\sum_{\substack{S \subseteq N \\
i \in S}} \frac{(k-1) !(n-k) !}{n !} \times \\
& \quad \times\left(V\left(S, x^{*}(t), t, T\right)-V\left(S \backslash\{i\}, x^{*}(t), t, T\right)\right),
\end{aligned}
$$

where $i=1, \ldots, n, k=|S|$.

Let there exist a solution set $\left\{Z_{i}^{S}, i \in N_{S}, t \geqslant t_{0}\right\}$ to the matrix Riccati differential equations (13) for every coalition $S \subset N$. Then we can calculate Shapley vector (24) where $V\left(S, x^{*}(t), t, T\right)$ is calculated according to (18). 


\section{Conclusion}

The concept of cooperative strategies for the class of differential games with continuous updating is presented. Cooperative strategies in linear-quadratic differential games with continuous updating are constructed and the corresponding theorem is presented. The characteristic function for the cooperative case is constructed, the path of possible solutions (e.g. Shapley value) based on it is indicated.

\section{References}

Basar, T. and G. Olsder (1995). Dynamic noncooperative game theory. Academic Press: London.

Bellman, R. (1957). Dynamic Programming. Princeton University Press: Princeton.

Engwerda, J. (2005). LQ Dynamic Optimization and Differential Games. Willey: New York.

Kleimenov, A. (1993). Non-antagonistic positional differential games. Science: Ekaterinburg.

Kuchkarov, I. and O. Petrosian (2019). On Class of Linear Quadratic Non-cooperative Differential Games with Continuous Updating. In: Mathematical Optimization Theory and Operations Research. MOTOR 2019. Lecture Notes in Computer Science (Khachay, M., Y. Kochetov, P. Pardalos eds) Lecture Notes in Computer Science, Vol. 11548, 635650. Springer: Cham

Petrosian, O.L. (2016). Looking Forward Approach in Cooperative Differential Games. International Game Theory Review, 18(2), 1-14.

Petrosian, O. L. (2016). Looking Forward Approach in Cooperative Differential Games with infinite-horizon. Vestnik S.-Petersburg Univ. Ser. 10. Prikl. Mat. Inform. Prots. Upr., 4, 18-30.

Petrosian, O. and A. Tur (2019). Hamilton-Jacobi-Bellman Equations for Non-cooperative Differential Games with Continuous Updating. In: Mathematical Optimization Theory and Operations Research. MOTOR 2019. Communications in Computer and Information Science (Bykadorov, I., V. Strusevich, T. Tchemisova eds.), Vol. 1090, 178-191. Springer: Cham

Petrosyan, L. and N. Murzov (1966). Game-theoretic problems in mechanics. Lithuanian Mathematical Collection, 3, 423-433.

Pontryagin, L. (1966). On the theory of differential games. Successes of Mathematical Sciences, 4(130), 219-274.

Shevkoplyas, E. (2014). Optimal solutions in differential games with random duration. Journal of Mathematical Sciences, 199(6), 715-722. 